Article

\title{
On the Effect of Electron Streaming and Existence of Quasi-Solitary Mode in a Strongly Coupled Quantum Dusty Plasma-Far and Near Critical Nonlinearity
}

\author{
Shatadru Chaudhuri and Asesh Roy Chowdhury *(D)
}

check for updates

Citation: Chaudhuri, S.; Chowdhury, A.R. On the Effect of Electron Streaming and Existence of Quasi-Solitary Mode in a Strongly Coupled Quantum Dusty

Plasma-Far and Near Critical Nonlinearity. Plasma 2021, 4, 408-425. https: / / doi.org/10.3390/plasma 4030030

Academic Editor: Andrey Starikovskiy

Received: 16 July 2021

Accepted: 23 August 2021

Published: 26 August 2021

Publisher's Note: MDPI stays neutral with regard to jurisdictional claims in published maps and institutional affiliations.

Copyright: (c) 2021 by the authors. Licensee MDPI, Basel, Switzerland. This article is an open access article distributed under the terms and conditions of the Creative Commons Attribution (CC BY) license (https:/ / creativecommons.org/licenses/by/ $4.0 /)$.
Department of Physics, Jadavpur University, Kolkata 700032, India; shatadru.chaudhuri12@gmail.com

* Correspondence: arc.roy@gmail.com

\begin{abstract}
A strongly coupled quantum dusty plasma consisting of electrons and dust with the ions in the background is considered when there is streaming of electrons. It is observed that the streaming gives rise to both the slow and fast modes of propagation. The nonlinear mode is then analyzed by the reductive perturbation approach, resulting in the KdV-equation. In the critical situation where non-linearity vanishes, the modified scaling results in the MKdV equation. It is observed that both the $\mathrm{KdV}$ and $\mathrm{MKdV}$ equations possess quasi-solitary wave solution, which not only has the character of a soliton but also has a periodic nature. Such types of solitons are nowadays called nanopteron solitons and are expressed in terms of cnoidal-type elliptic functions.
\end{abstract}

Keywords: quasi-soliton; quantum plasma; dusty plasma; strongly coupled plasma

\section{Introduction}

The interest in study of plasmas containing dust particles are growing day by day, because of the importance of such plasmas in the study of the space environment, such as asteroid zones, planetary rings, cometary tails as well as lower ionosphere of earth. The dust-in-plasma or the dusty plasma contain dust grains, which has a dimension of the order of micrometer to sub-micrometer [1-3].

Quantum aspects of plasma gained importance in recent times due to its application in plasma echoes [4] and micro-electronic devices [5]. Initially the subject was formulated and studied by Haas [6] and his collaborators [7]. It has applications in Fermi gas [8], quantum Penrose diagrams and quantum plasma instabilities [9]. Moreover, the quantum hydrodynamic model deals only with the macroscopic variables, namely the density and velocity stress tensors, etc., and also includes the so called Bohm potential [10]. On the other hand a different approach of quantum plasma is that of Wigner-Poisson equation, which is actually a kinetic model [11]. However, it is less effective in numerical simulations compared to that of the Q.H.D. (Quantum Hydrodynamic Model).

Actually, the field of quantum plasma physics has a long and diverse tradition and is garnering increasing interest that is motivated by its potential applications in modern technology. The quantum degeneracy effects start playing a significant role when the de Broglie thermal wavelength $\lambda=\frac{\hbar}{\sqrt{2 \pi m_{e} k_{B} T}}$ for electrons is similar to or larger than the average interelectron distance ' $n_{e}^{1 / 3}$ '. Moreover, one can show that when the temperature approaches the electron Fermi temperature $T_{F e}$, the equilibrium distribution function changes from the Maxwell-Boltzmann $\propto \exp \left(-E / k_{B} T\right)$ to the Fermi-Dirac one.

Another important domain of present day plasma physics is the study of dusty plasma [12], and thus many analyses have been performed on quantum dusty plasma [13,14]. The emergence of the dust acoustic wave (D.A.W) is an important aspect of such analysis [15]. Dust Acoustic Waves (DAW) have been discussed in connection with various structures that have been observed in Saturn rings, such as spokes, braids and clumps [16]. The study of quantum plasma has been extensively performed by Haas [17], Shukla [18] 
and many others. However, in the domain of dusty plasma, the important phenomenon is of strong coupling of dust particles [19]. The corresponding investigation has been performed at least in two main formulations: one is to use the visco-elastic model [20] and the other by using effective electrostatic pressure [21]. It was Ikezi [22] who predicted that a dusty plasma can enter the strong coupling regime due to high charge and low temperature of the dust that makes the coupling co-efficient $\Gamma \gg 1$, and the coupling co-efficient $\Gamma=\frac{e^{2} Z_{d}^{2}}{4 \pi \varepsilon_{0} k_{B} T_{d} a_{d}}$ is the ratio between the Coulomb potential energy and thermal energy used to measure the relative importance of these electrostatic forces on the dust grain. Here ' $T_{d}$ ', ' $Z_{d}$ ' and ' $a_{d}=n_{d}^{1 / 3 \text { ' }}$ are the temperature, charge number and mean inter-particle distance of the dust, respectively. The equation of the state of strongly coupled plasma was formulated by Cousens et al. [23] and written as $P^{*}=n_{d} k_{B} T^{*}$, the effective electrostatic pressure. ' $T$ ' is the effective electrostatic temperature defined as $k_{B} T^{*}=\frac{N_{n n} e^{2} Z_{d}^{2}}{12 \pi \varepsilon_{0}} \sqrt[3]{n_{d}}(1+\mathcal{K}) \exp (-\mathcal{K})$ where ' $N_{n n}$ ' is the number of nearest neighbors determined by the normalized inter-particle distance.

On the other-hand, quantum dusty plasma [22] is well known as the feature of high charge and low temperature of dust grains with high number density compared with that of classical plasma, which renders the existence of strongly coupled regimes appearing in quantum plasma possible.

In the first part, we analyzed the linear mode of propagation by starting with the normalized electron-dust-ion equation, where we have assumed that the electrons possess a streaming velocity. The immediate consequence is that we obtain a fast and slow mode of propagation in the dusty plasma. In the next section we applied the reductive perturbation scheme in order to deduce the KdV equation, and it is found that we have a new kind of nonlinear excitation, which is a soliton having a periodic tail. In the next part, we consider the critical case when the nonlinearity coefficient tends to zero and the usual modification of the reductive computation results in the MKdV situation. Such an equation is also observed to posses a cnoidal-type soliton.

\section{Formulation}

Let us consider a plasma where ' $n_{e}$ ' is the electron density, ' $u_{e}$ ' is the corresponding velocity and the same quantities for the dust particle are denoted as ' $n_{d}{ }^{\prime}$, ' $u_{d}$ '. The mass of electron and dust are denoted as ' $m_{e}{ }^{\prime}$, ' $m_{d}$ ' respectively. If these are in motion then there will be an electrostatic potential ' $\phi$ ' and pressure ' $P_{d}$ '. The Quantum Hydrodynamic (QHD) equations are as follows:

$$
\begin{gathered}
\frac{\partial n_{e}}{\partial t}+\frac{\partial}{\partial x}\left(n_{e} u_{e}\right)=0 \\
\frac{\partial u_{e}}{\partial t}+u_{e} \frac{\partial u_{e}}{\partial x}=\frac{\hbar^{2}}{2 m_{e}^{2}} \frac{\partial}{\partial x}\left\{\frac{\partial^{2} / \partial x^{2}\left(\sqrt{n_{e}}\right)}{\sqrt{n_{e}}}\right\}+\frac{e}{m_{e}} \frac{\partial \phi}{\partial x} \\
\frac{\partial n_{d}}{\partial t}+\frac{\partial}{\partial x}\left(n_{d} u_{d}\right)=0 \\
\frac{\partial u_{d}}{\partial t}+u_{d} \frac{\partial u_{d}}{\partial x}=\frac{\hbar^{2}}{2 m_{d}^{2}} \frac{\partial}{\partial x}\left\{\frac{\partial^{2} / \partial x^{2}\left(\sqrt{n_{d}}\right)}{\sqrt{n_{d}}}\right\}-\frac{e}{m_{d}} \frac{\partial \phi}{\partial x}-\frac{\partial P_{d}}{\partial x} \\
\frac{\partial^{2} \phi}{\partial x^{2}}=\frac{e}{\varepsilon_{0}}\left(n_{e}+n_{d}-n_{0}\right)
\end{gathered}
$$

' $\phi$ ' is the electrostatic potential here. Moreover, we consider the ions to be immobile and to have a distribution of the following form.

$$
n_{0}=n_{i 0} \exp \left(-\frac{e \phi}{k_{B} T_{i}}\right)
$$


The normalized set of equation governing the dusty plasma can be written as follows:

$$
\begin{gathered}
\frac{\partial n_{e}}{\partial t}+\frac{\partial}{\partial x}\left(n_{e} u_{e}\right)=0 \\
\frac{\partial u_{e}}{\partial t}+u_{e} \frac{\partial u_{e}}{\partial x}=H^{2} \alpha_{1} \frac{\partial}{\partial x}\left\{\frac{\partial^{2} / \partial x^{2}\left(\sqrt{n_{e}}\right)}{\sqrt{n_{e}}}\right\}+\frac{\alpha_{1}}{Z_{d}} \frac{\partial \phi}{\partial x} \\
\frac{\partial n_{d}}{\partial t}+\frac{\partial}{\partial x}\left(n_{d} u_{d}\right)=0 \\
\frac{\partial u_{d}}{\partial t}+u_{d} \frac{\partial u_{d}}{\partial x}=H^{2} \alpha_{1} \frac{\partial}{\partial x}\left\{\frac{\partial^{2} / \partial x^{2}\left(\sqrt{n_{d}}\right)}{\sqrt{n_{d}}}\right\}-\frac{\partial \phi}{\partial x}-\frac{1}{Z_{d}} \frac{\partial\left(n_{d} d\right)}{\partial x} \\
\frac{\partial^{2} \phi}{\partial x^{2}}=\mu_{1} n_{e}+n_{d}-\beta \exp (-S \phi)
\end{gathered}
$$

where the normalized distribution for the background ions is as follows

$$
n_{0}=\beta \exp (-S \phi)
$$

with $\mu_{1}=\frac{n_{e 0}}{Z_{d} n_{d 0}}, \beta=\frac{n_{i 0}}{Z_{d} n_{d 0}}$ and $S=\frac{T_{0}}{T_{i}}$ where ' $n_{i 0}$ ' and ' $n_{e 0}$ ' are, respectively, the ionelectron equilibrium densities, and ' $T_{i}$ ' and ' $T_{e}$ ' are their temperatures with $T_{0}=\frac{Z_{d}^{2} n_{d 0} T_{i} T_{e}}{n_{i 0} T_{e}+n_{e 0} T_{i}}$, where we have used all the scaled variables. Here ' $u_{e}$ ' and ' $n_{e}$ ' are the velocity and density of electrons, ' $u_{d}$ ', ' $n_{d}$ ' are those of the dust grains and ' $\phi$ ' is the electrostatic potential. The strong interaction between the dust grains are expressed as a force given by the potential.

The normalized variables are defined as $t \rightarrow \omega_{p d} t ; x \rightarrow x / \lambda_{D} ; u_{d} \rightarrow u_{d} / C_{d}$; $u_{e} \rightarrow u_{e} / C_{d} ; n_{d} \rightarrow n_{d} / n_{d 0} ; n_{e} \rightarrow n_{e} / n_{e 0} ;$ and $\phi \rightarrow \phi /\left(\frac{k_{B} T_{0}}{e}\right)$, where $\omega_{p d}=\sqrt{\frac{Z_{d}^{2} e^{2} n_{d 0}}{\epsilon_{0} m_{d}}}$; $\lambda=\sqrt{\frac{\epsilon_{0} k_{B} T_{0}}{Z_{d}^{2} n_{d 0} e^{2}}}$; and $C_{d}=\sqrt{\frac{Z_{d} k_{B} T_{0}}{m_{d}}}$.

\section{Linear Propagation and Streaming}

In order to analyse the linear propagation mode, we set the following:

$$
\left.\begin{array}{r}
n_{e}=1+n_{e 1} \exp [i(k x-\omega t)] \\
u_{e}=u_{e 0}+u_{e 1} \exp [i(k x-\omega t)] \\
n_{d}=1+n_{d 1} \exp [i(k x-\omega t)] \\
u_{d}=u_{d 1} \exp [i(k x-\omega t)] \\
\phi=\phi_{1} \exp [i(k x-\omega t)] \\
d=d_{0}+\epsilon d_{1}
\end{array}\right\}
$$

where $d_{1}=d_{11} n_{d 1}+d_{12} \phi_{1}$ (from Reference [23]) provided that, at equilibrium, $Z_{d} n_{e 0}+$ $n_{d 0}=Z_{d} n_{i 0}$. Linearizing the set (7)-(12) results in the following.

$$
\begin{aligned}
& n_{e 1}=\frac{k}{\omega-k u_{e 0}} u_{e 1} \\
& u_{e 1}=\left\{\frac{k Z_{d} \alpha_{1}}{\left(\omega-k u_{e 0}\right)\left[1-\frac{H^{2} k^{4}}{2} \frac{\alpha_{1}}{\left(\omega-k u_{e 0}\right)^{2}}\right]}\right\} \phi_{1} \\
& n_{d 1}=\frac{k}{\omega} u_{d 1} \\
& u_{d 1}=-\frac{\left(d_{12}+1\right)\left(1 / Z_{d}\right)(k / \omega)}{\left[1-\frac{H^{2} k^{4}}{2}+\frac{1}{Z_{d}}\left(d_{0}+d_{11}\right) \frac{k^{2}}{\omega^{2}}\right]} \phi_{1}
\end{aligned}
$$




$$
-k^{2} \phi_{1}=\mu_{1} n_{e 1}+n_{d 1}+\beta \phi_{1}
$$

These relations along with the equilibrium condition results in the following dispersion relation.

$$
\begin{array}{r}
\left(k^{2}+S \beta\right)+\frac{\mu_{1} Z_{d} \alpha_{1} k^{2}}{\left[\left(\omega-k u_{e 0}\right)^{2}-\frac{H^{2} k^{4} \alpha_{1}}{2}\right]}-\frac{k^{2}\left(d_{12}+1\right)}{Z_{d}} \times \\
\frac{1}{\left[\omega^{2}-\frac{H^{2} k^{4} \alpha_{1}}{2}+\frac{\left(d_{0}+d_{11}\right) k^{2}}{Z_{d}}\right]}=0
\end{array}
$$

Equation (19) can also be written as follows:

$$
\omega^{4}+a \omega^{3}+b \omega^{2}+c \omega+d=0
$$

where $a=-\frac{2 k u_{e 0}}{\left(k^{2}+S \beta\right)} ; b=\left(-\Gamma_{2} k^{4}+\Gamma_{3} k^{2}-\Gamma_{1} k^{4}+k^{2} u_{e 0}^{2}-\Gamma_{5} k^{2}\right) ; c=\left[\left(k^{2}+S \beta\right)\left(2 k^{5} u_{e 0} \Gamma_{2}-\right.\right.$ $\left.\left.2 k u_{e 0} \Gamma_{3} k^{2}\right)-\Gamma_{5} k^{3} u_{e 0}\right] /\left(k^{2}+S \beta\right) ; d=\left[\left(k^{2}+S \beta\right)\left(-k^{6} u_{e 0}^{2} \Gamma_{2}+\Gamma_{3} u_{e 0}^{2} k^{4}+\Gamma_{1} \Gamma_{2} k^{8}-\Gamma_{1} \Gamma_{3} k^{6}\right)-\right.$ $\left.\Gamma_{4} k^{2}\left(\Gamma_{2} k^{4}+\Gamma_{3} k^{2}\right)-\Gamma_{5} k^{2}\left(k^{2} u_{e 0}^{2}-\Gamma_{1} k^{4}\right)\right] /\left(k^{2}+S \beta\right)$.

We also have the following: $\Gamma_{1}=\frac{H^{2} k^{4} \alpha_{1}}{2} ; \Gamma_{2}=\frac{H^{2} k^{4} \alpha_{1}}{2} ; \Gamma_{3}=\frac{d_{0}+d_{11} k^{4}}{Z_{d}} ; \Gamma_{4}=\mu_{1} Z_{d} \alpha_{1} k^{2}$; $\frac{1}{Z_{d}}\left(d_{12}+1\right) k^{2}$.

The solution of the quartic Equation (20) is as follows:

$$
\left.\begin{array}{l}
\omega_{1,2}=-\frac{1}{2}\left(\frac{a}{2}+B\right) \pm \frac{1}{2} \sqrt{\left(\frac{a}{2}+B\right)^{2}-4(A+E)} \\
\omega_{3,4}=-\frac{1}{2}\left(\frac{a}{2}-B\right) \pm \frac{1}{2} \sqrt{\left(\frac{a}{2}-B\right)^{2}-4(A-E)}
\end{array}\right\}
$$

where the following is the case.

$A=\frac{b}{3}+\left(\frac{q}{2} \pm \frac{1}{2} \sqrt{q^{2}-\frac{4}{27} p^{3}}\right)^{1 / 3}+\left(\frac{q}{2} \mp \frac{1}{2} \sqrt{q^{2}-\frac{4}{27} p^{3}}\right)^{1 / 3}$

$p=3 \chi^{2}-2 \chi b+\theta ; q=\chi^{3}-b \chi^{2}+\theta \chi+\lambda ; \chi=b / 3 ; \theta=(1 / 4)(2 c a-4 d) ;(1 / 4)(b d-$ $\left.a^{2} d-c^{2}\right) ; B=\sqrt{\frac{a^{2}}{4}+A-b} ; E=\sqrt{A^{2}-d}$

From the equation set (21), one can observe that ' $\omega_{1}$ ' and ' $\omega_{3}$ ' are physically admissible of the one that gives the slow mode and the other that gives the fast mode. In order to obtain an idea of the physical situation, we have graphically plotted the linear dispersion relation in Figures 1-8. In Figure 1 we show the case where there is no streaming or ' $u_{e 0}=0$ ' and the effect of strong coupling is also absent, while in Figure 2 we have shown the situation where there is no streaming but the strong coupling effect is present and as a result the nature of variation of the dispersion relation changes. Moreover due to the presence of strong coupling the wave frequency also gets increased.

One should note that in both the Figures 1 and 2, we observe that the quantum diffraction parameter ' $H$ ' has a very prominent effect. Next, in the Figures 3 and 4 , we have shown the variation of the wave frequency with the streaming velocity ' $u_{e 0}$ ' both in the presence and absence of strong coupling effect.

In the Figures 5-8, we show how the electron streaming gives rise to two modes, namely 'slow' and 'fast', and how they vary with the diffraction parameter ' $\mathrm{H}$ ', streaming velocity ' $u_{e 0}$ ' and the effect of strong coupling. 


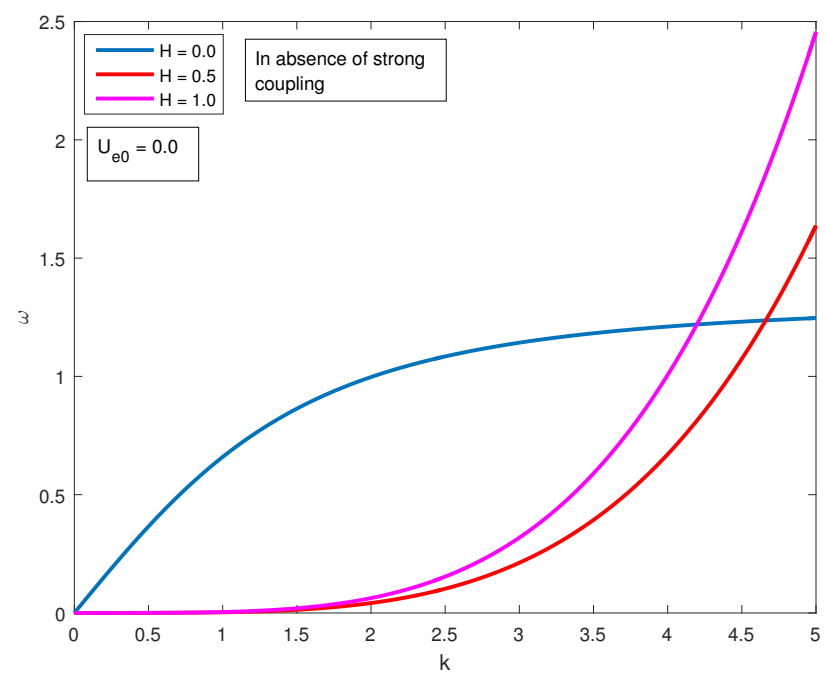

Figure 1. The plot of the dispersion relation in the absence of strong coupling and electron streaming velocity.

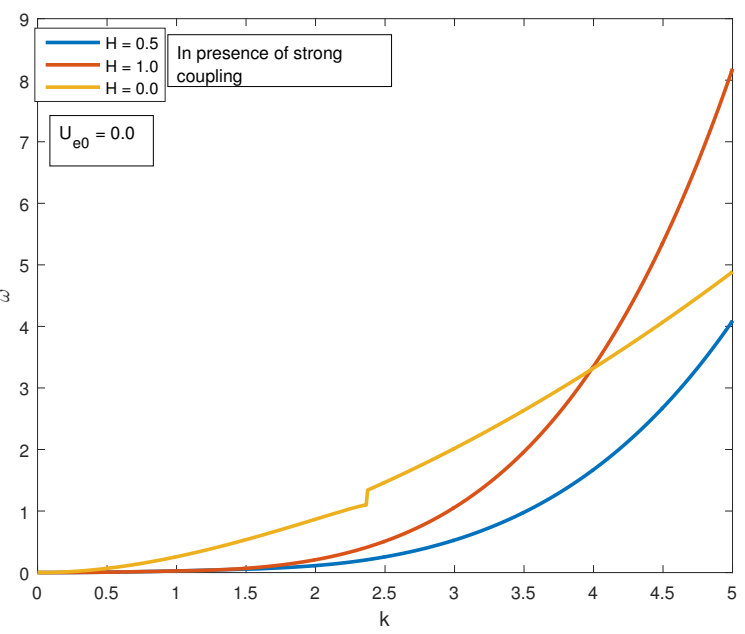

Figure 2. The plot of the dispersion relation in the presence of strong coupling but in the absence of electron streaming velocity.

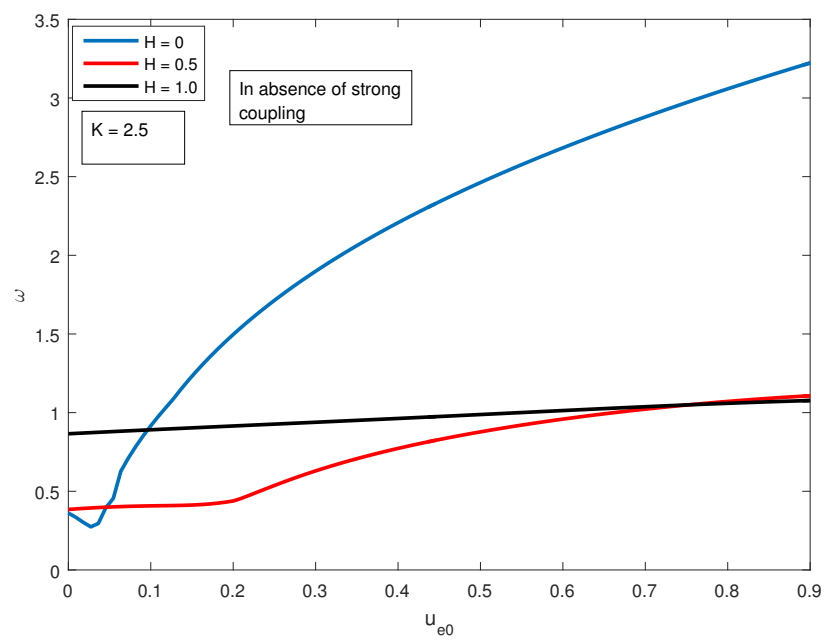

Figure 3. The variation of the wave frequency with the electron streaming velocity. In this case, we see that the wave frequency varies quite clearly with the quantum parameter ' $H$ '. 


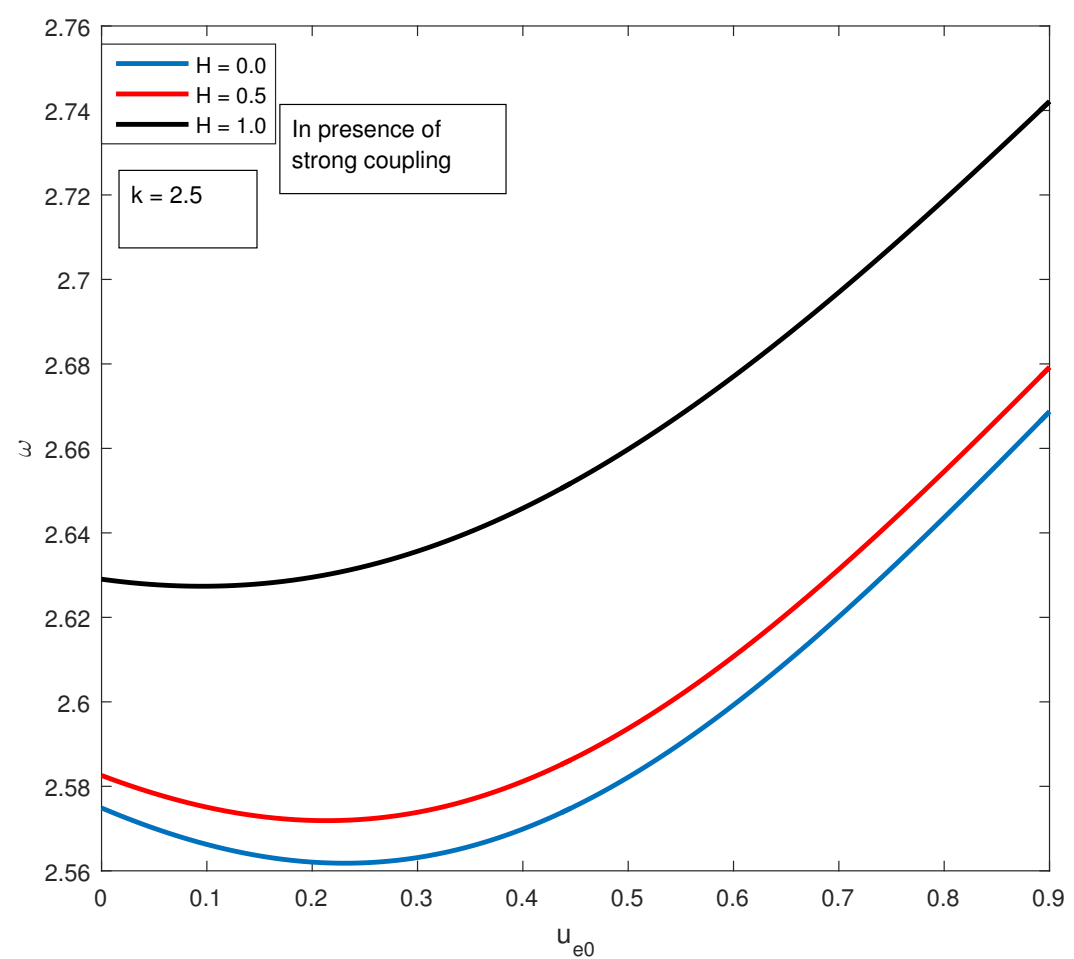

Figure 4. The variation of the wave frequency with the electron streaming velocity. In this case, we see that the wave frequency varies quite clearly with the quantum parameter ' $H$ '.

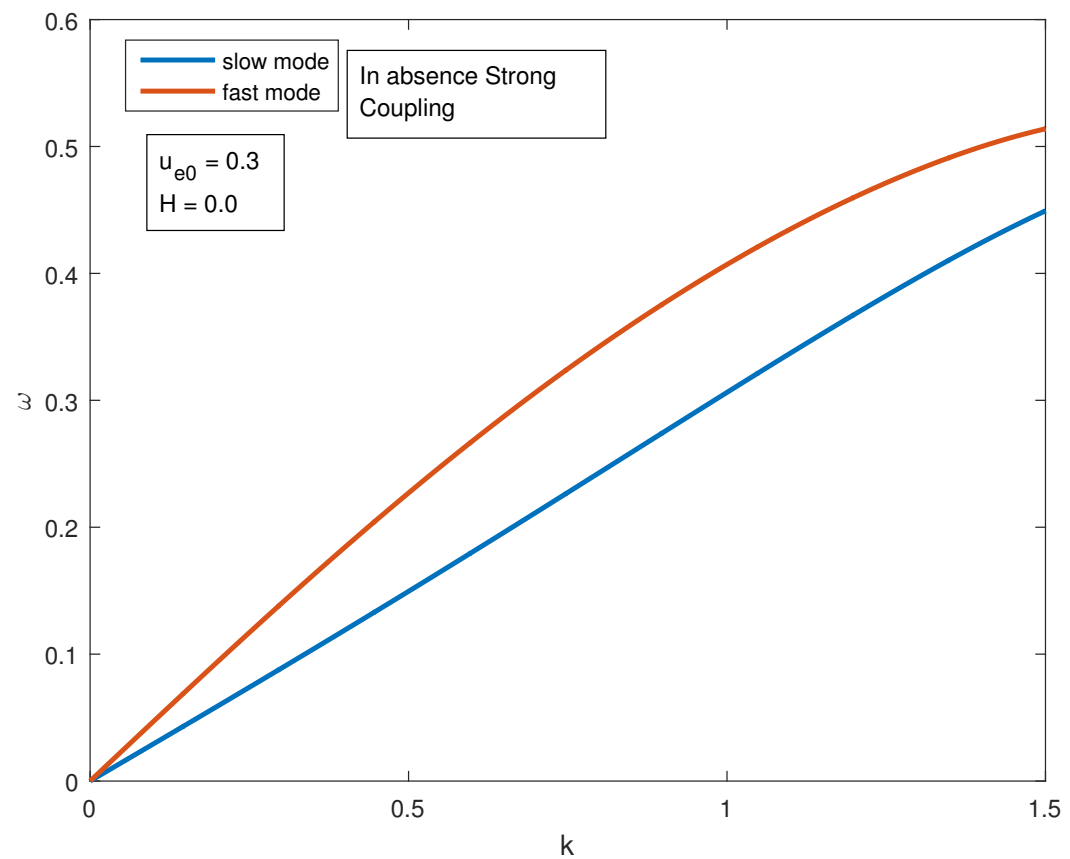

Figure 5. The figure depicts the 'fast' and 'slow' modes of excitation in absence of strong coupling and for the quantum situation. 


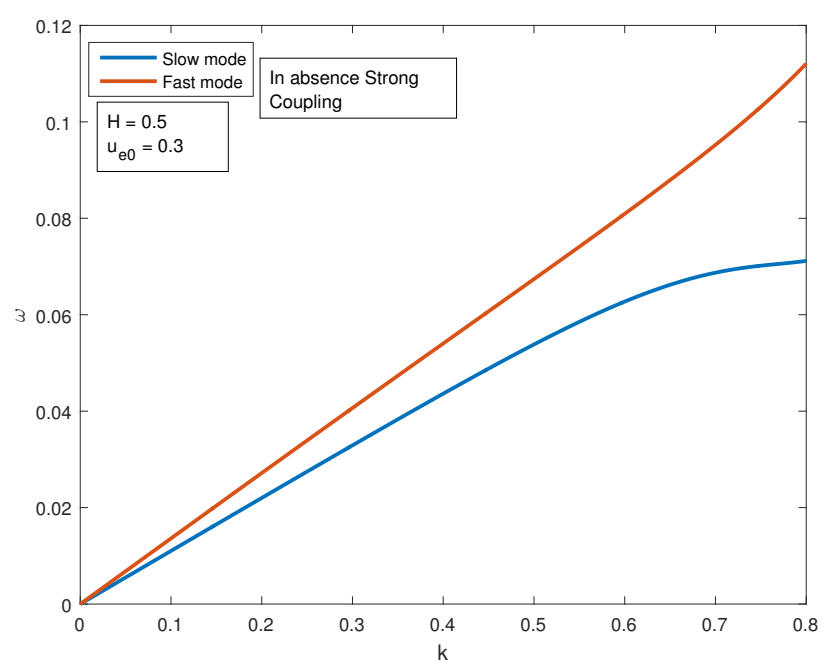

Figure 6. The figure depicts the 'fast' and 'slow' modes of excitation in absence of strong coupling but for the classical situation.

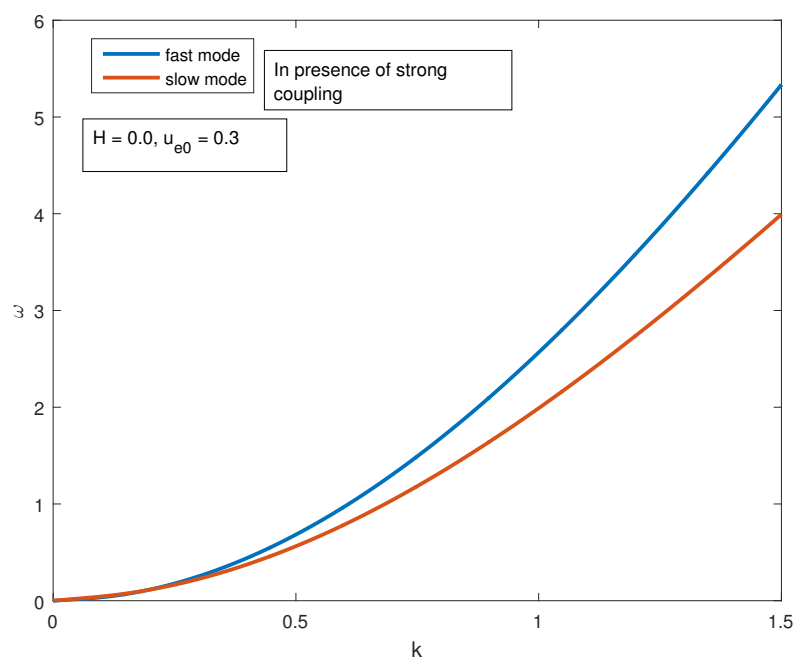

Figure 7. The figure depicts the 'fast' and 'slow' modes of excitation in presence of strong coupling but for the classical situation.

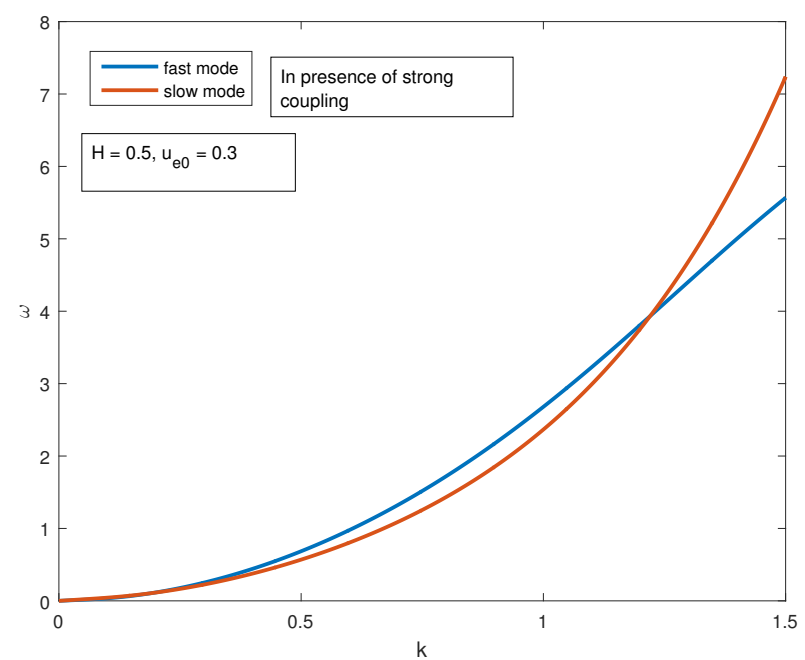

Figure 8. The figure depicts the 'fast' and 'slow' modes of excitation in presence of strong coupling in the quantum situation. 


\section{Reductive Perturbation Approach and the Derivation of the Kdv Equation}

In order to analyze the nonlinear propagating mode of the system, we adapt the reductive approach and set the following:

$$
\left.\begin{array}{r}
\xi=\epsilon^{1 / 2}(x-\lambda t) \\
\tau=\epsilon^{3 / 2} t
\end{array}\right\}
$$

along with

$$
\left.\begin{array}{r}
n_{e}=1+\epsilon n_{e 1}+\epsilon^{2} n_{e 2}+\epsilon^{3} n_{e 3}+\cdots \\
u_{e}=u_{e 0}+\epsilon u_{e 1}+\epsilon^{2} u_{e 2}+\epsilon^{3} u_{e 3}+\cdots \\
n_{d}=1+\epsilon n_{d 1}+\epsilon^{2} n_{d 2}+\epsilon^{3} n_{d 3}+\cdots \\
u_{d}=\epsilon u_{d 1}+\epsilon^{2} u_{d 2}+\epsilon^{3} u_{d 3}+\cdots \\
\phi=\epsilon \phi_{1}+\epsilon^{2} \phi_{2}+\epsilon^{3} \phi_{3}+\cdots \\
d=d_{0}+\epsilon d_{1}+\epsilon^{2} d_{2}+\epsilon^{3} d_{3}+\cdots
\end{array}\right\}
$$

whence in lowest order of $\epsilon$ we obtain the following.

$$
\left.\begin{array}{r}
n_{e 1}=\frac{u_{e 1}}{\left(\lambda-u_{e 0}\right)} \\
n_{d 1}=\frac{u_{e 1}}{\lambda} \\
u_{e 1}=-\frac{\alpha_{3}}{Z\left(\lambda-u_{e 0}\right)} \phi_{1} \\
u_{d 1}=-\frac{\left(d_{12}+1\right) Z}{Z \lambda^{2}-\left(d_{0}+d_{11}\right)} \phi_{1} \\
n_{d 1}+\mu_{1} n_{e 1}+S \beta \phi_{1}=0
\end{array}\right\}
$$

This results in the dispersion relation for the long wavelength situation.

$$
-\frac{\alpha_{1} \mu_{1}}{Z_{d}\left(\lambda-u_{e 0}\right)^{2}}+\frac{\left(d_{12}-1\right)}{\left[\lambda^{2}-\left(d_{0}+d_{11}\right)\right]}+S \beta=0
$$

In the next order of $\epsilon$, that is, from the terms of $\epsilon^{5 / 2}$, we obtain the following:

$$
\begin{gathered}
\frac{\partial n_{e 1}}{\partial \tau}-\lambda \frac{\partial n_{e 2}}{\partial \xi}+\frac{\partial u_{e 2}}{\partial \xi}+\frac{\partial}{\partial \xi}\left(n_{e 1} u_{e 1}\right)+u_{e 0} \frac{\partial n_{e 2}}{\partial \xi}=0 \\
\frac{\partial n_{d 1}}{\partial \tau}-\lambda \frac{\partial n_{d 2}}{\partial \xi}+\frac{\partial u_{d 2}}{\partial \xi}+\frac{\partial}{\partial \xi}\left(n_{d 1} u_{d 1}\right)=0 \\
\frac{\partial u_{e 1}}{\partial \tau}-\lambda \frac{\partial u_{e 2}}{\partial \xi}+u_{e 0} \frac{\partial u_{e 2}}{\partial \xi}+u_{e 1} \frac{\partial u_{e 1}}{\partial \xi}=\alpha_{1} H^{2} \frac{\partial^{3} n_{e 1}}{\partial \xi^{3}}+\frac{\alpha_{3}}{Z} \frac{\partial \phi_{2}}{\partial \xi} \\
\frac{\partial u_{d 1}}{\partial \tau}-\lambda \frac{\partial u_{d 2}}{\partial \xi}+u_{e 1} \frac{\partial u_{e 1}}{\partial \xi}=\alpha_{1} H^{2} \frac{\partial^{3} n_{d 1}}{\partial \xi^{3}}- \\
\frac{1}{Z} \frac{\partial}{\partial \xi}\left(d_{0} n_{d 2}\right)-\frac{\partial \phi_{2}}{\partial \xi}-\frac{1}{Z} \frac{\partial d_{2}}{\partial \xi}-\frac{1}{Z} \frac{\partial}{\partial \xi}\left(n_{d 1} d_{1}\right) \\
\frac{\partial^{2} \phi_{1}}{\partial \xi^{2}}=n_{d 2}+\mu_{1} n_{e 2}-S \beta \phi_{2}+\beta S^{2} \phi_{1}^{2}
\end{gathered}
$$

If we eliminate all higher order terms, we obtain the $\mathrm{KdV}$ equation:

$$
\frac{\partial \phi_{1}}{\partial \tau}+A \phi \frac{\partial \phi_{1}}{\partial \xi}+B \frac{\partial^{3} \phi_{1}}{\partial \xi^{3}}=0
$$

with $A=A_{1} / A_{2}$ and $B=B_{1} / A_{2}$, where ' $A_{1}$ ', ' $A_{2}$ ' and ' $B_{1}$ ' are given in Appendix A. The expression of ' $d_{2}$ ' in Equation (29) is given by $d_{2}=d_{21} n_{2}+d_{22} \phi_{2}+d_{23} n_{1}^{2}+d_{24} n_{1} \phi_{1} d_{25} \phi_{1}^{2}$, where the constants $d_{21}$ and $d_{22}$, etc., are given as per Reference [23]. 


\subsection{Soliton Cnoidal Wave Solution}

It is well known that the KdV-equation wrote above always supports stationary wave solutions. However, recent studies have revealed a whole new class of solutions for the $\mathrm{KdV}$ problem. It may be mentioned that Boyd observed that one can numerically obtain a new class of solutions that does not follow the definition of soliton in the very strict sense, which is in the sense that it has a long tail that is a periodic waveform. Such novel kinds of patterns are named the 'nanopteron' solitons. These are a wide class known as the quasi-soliton. Later on, it was found that one can obtain the analytic form of such a solution by using the famous 'tanh'-expansion method.

Let us set the following:

$$
\phi_{1}=u_{0}+u_{1} \tanh (w)+u_{2} \tanh (w)^{2}
$$

where, $u_{0}, u_{1}, u_{2}$ and $w$ are functions of $(x, t)$ to be determined. By substituting Equation (36) in (35) and equating various powers of $\tanh (w)$, a system of over-determined equations are obtained. It is really interesting to note that all these are consistent with one another. Finally, one obtains $u_{2}=-\frac{12 B w_{x}^{2}}{A}$ and $u_{1}=\frac{12 B w_{x x}}{A}$ :

$$
\frac{B}{A}\left(\frac{w_{x x x}}{w_{x}}-\frac{3 w_{x x}^{2}}{2 w_{x}^{2}}-2 w_{x}^{2}\right) \frac{w_{t}}{A w_{x}}
$$

along with the fact that $w(x, t)$ satisfies the following.

$$
\frac{w_{t}}{w_{x}}+B\left(\frac{w_{x x x}}{w_{x}}-\frac{3 w_{x x}^{2}}{2 w_{x}^{2}}-2 w_{x}^{2}\right)+\lambda=0
$$

This $\lambda$ is a constant of integration. This last equation actually possesses a wide variety of solutions of which we only consider the following one:

$$
w=\xi+c_{1} \tanh ^{-1}\left(c_{2} \operatorname{sn}(\eta, m)\right)
$$

with the following.

$$
\left.\begin{array}{l}
\xi=\frac{x-V_{1} t}{W_{1}} \\
\eta=\frac{x-V_{2} t}{W_{2}}
\end{array}\right\}
$$

' $s n^{\prime}$ ' is the Jacobi-elliptic function with ellipticity ' $m$ '. It is interesting to note that ' $V_{1}$ ' and ' $V_{2}$ ' are the velocities of the soliton and the surrounding cnoidal wave. Thus, we can finally write the solution for $\phi_{1}$ :

$$
\begin{array}{r}
\phi_{1}=\frac{3\left(V_{1}-V_{2}\right)}{2 A G^{2}}\left[\frac{\left(m^{2}-1+2 G\right)^{2}}{\left(m^{2}-1\right)} \tanh (w)^{2}-\right. \\
\left.2 \delta m S\left(m^{2}-1+2 G\right) \tanh (w)+\left(m^{2}-1\right)\right]- \\
\frac{\left(m^{2}+7\right) V_{1}-\left(3 m^{2}+5\right) V_{2}}{2 A\left(m^{2}-1\right)}
\end{array}
$$


with the following.

$$
\left.\begin{array}{r}
w=\frac{\xi-V_{1} \tau}{w_{1}}+c_{1} \tanh ^{-1}\left(c_{2} S(\eta, m)\right) \\
G=1-m^{2} S^{2}+\delta m C D \\
\eta=\frac{\xi-V_{2} \tau}{w_{2}} \\
S=\operatorname{sn}(\eta, m) ; C=c n(\eta, m) ; D=d n(\eta, m) \\
W_{1}=\sqrt{\frac{8 B\left(1-m^{2}\right)}{V_{1}-V_{2}}} \\
W_{2}=\sqrt{\frac{2 B\left(1-m^{2}\right)}{V_{1}-V_{2}}} \\
c_{1}=\delta / 2 ; c_{2}=m ; \delta^{2}=1
\end{array}\right\}
$$

It is now interesting to observe that for $m_{e}=9.1 * 10^{-31} \mathrm{~kg} ; m_{i}=6.7 * 10^{-26} \mathrm{~kg}$; $m_{d}=8 * 10^{-12} \mathrm{~kg} ; n_{e 0}=4 * 10^{13} \mathrm{~m}^{-3} ; n_{i 0}=7 * 10^{13} \mathrm{~m}^{-3} ; T_{i}=11,600$ kelvin; $T_{e}=10^{4}$ kelvin; $T_{e}=5 * 10^{4}$ kelvin; $T_{e}=8 * 10^{4}$ kelvin; $Z=4.3 * 10^{5} ; u_{e}=1.5$; $m=0.04 ; \delta=-1 ;$ and $V_{1}=0.05 ; V_{2}=-0.05$, the form of $\phi_{1}$ if plotted against $\xi$ turns out to be of the form given in Figure 9.

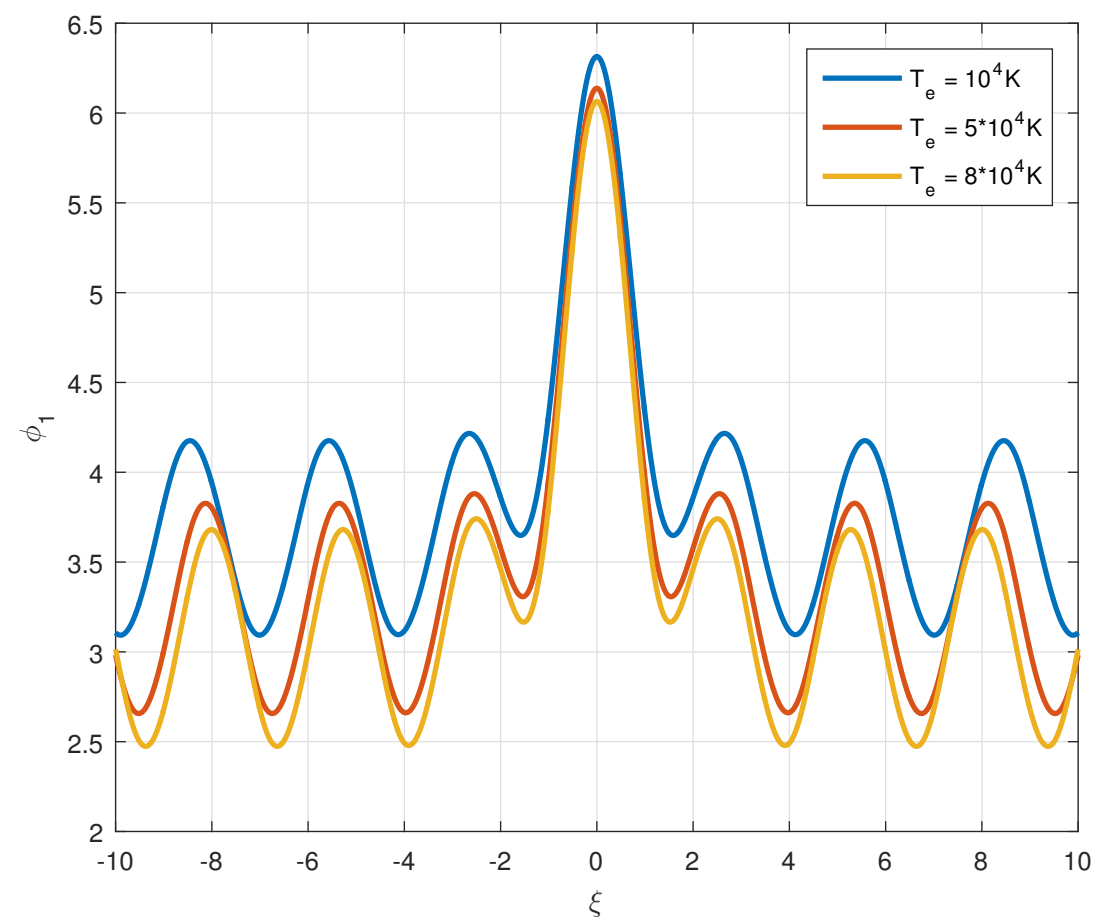

Figure 9. This is the form of quasi-soliton or the nanopteron soliton for which its analytical form is given by (40).

One may note that here we have a central solitary profile, but its tails are not approaching zero in either direction of $\xi$. Instead, we have purely sinusoidal behaviour such as $\xi \rightarrow \pm \infty$. We have shown how the form changes with the electron temperature $T_{e}$. On the other-hand, if one has $m=0$, then we obtain the purely solitary wave, as shown in the Figure 10. 


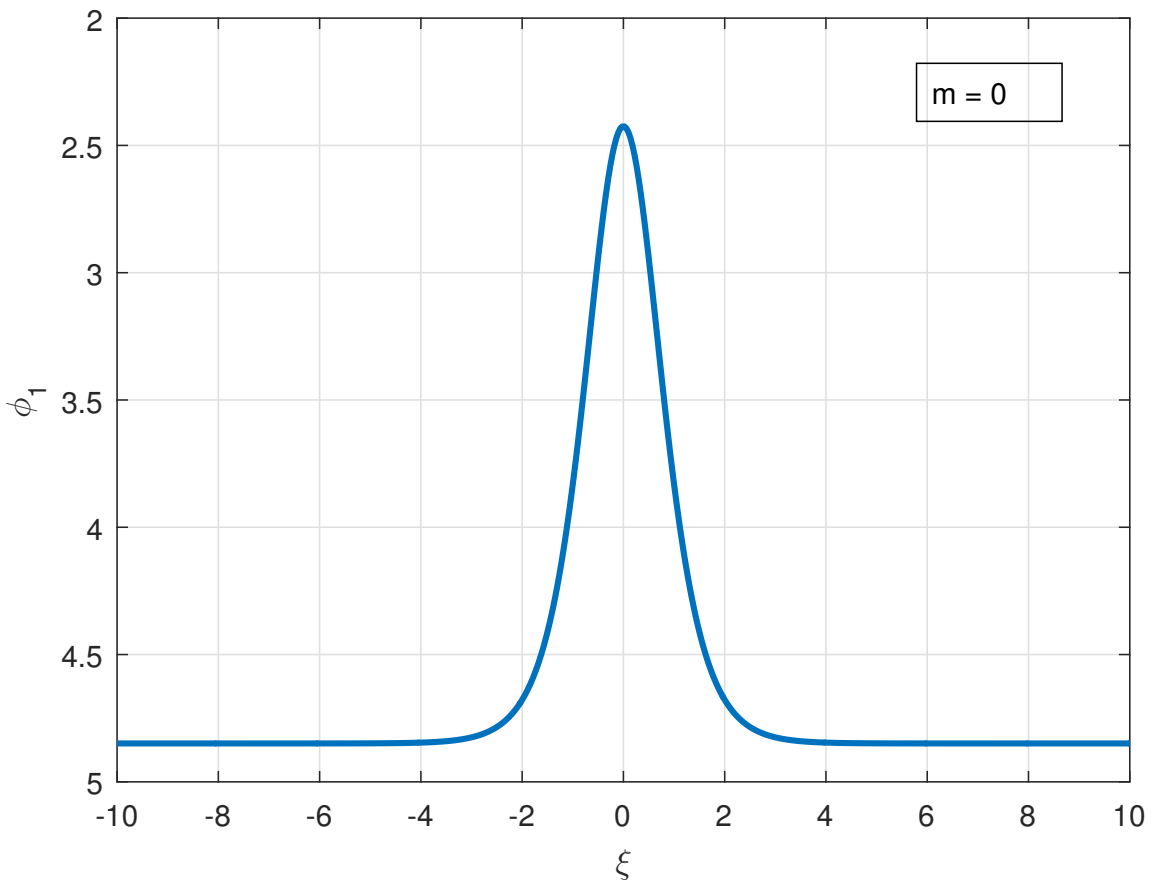

Figure 10. The quasi-soliton transforms to pure soliton for $m \rightarrow 0$.

Moreover, in the Figure 11 we show the three dimensional form of this new wave form. Lastly, in Figure 12, we show a contour plot of $\phi_{1}$ where the ordinate is time ' $\tau$ ' and the abscissa is ' $\mathcal{}$ ' for different parameter values.

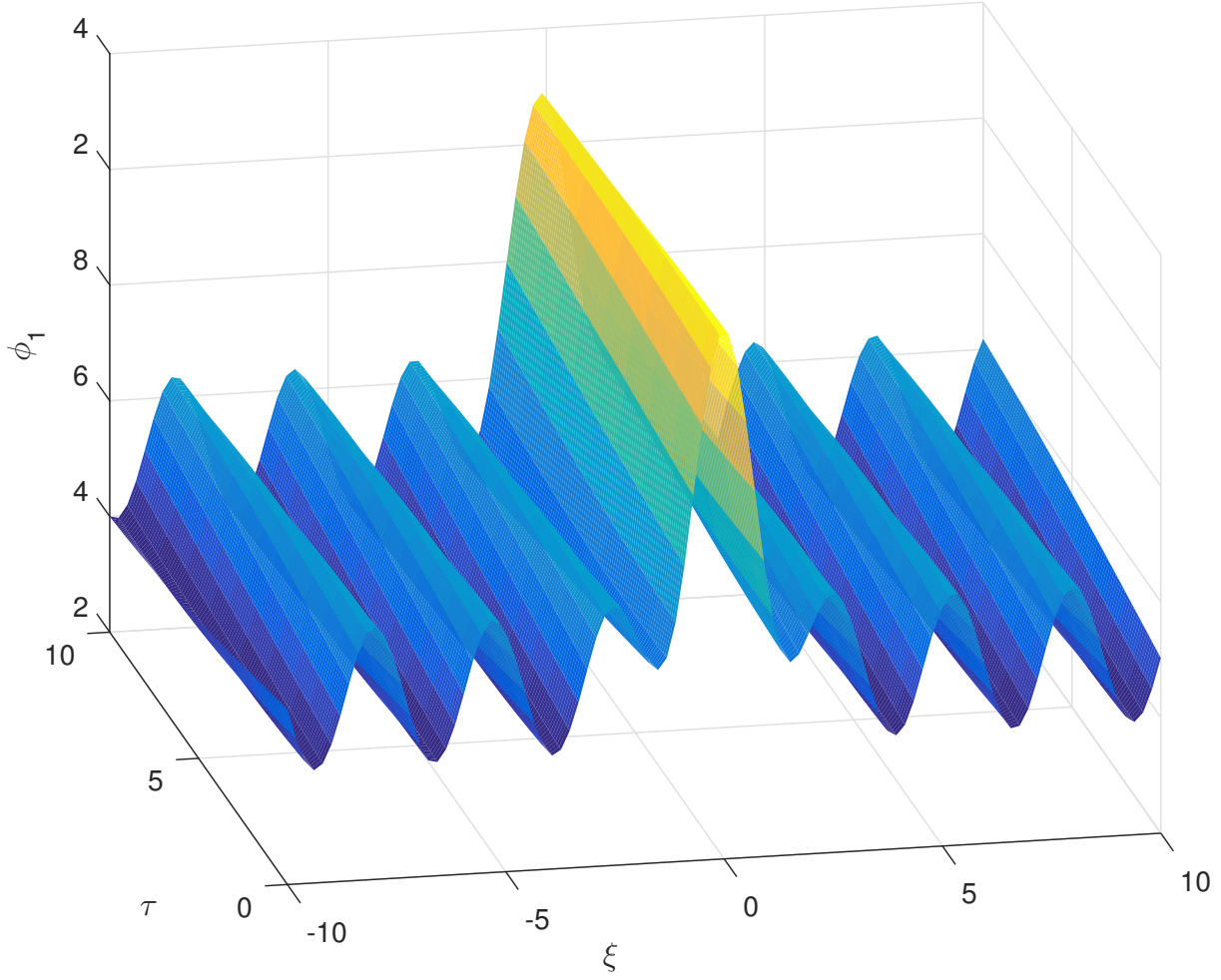

Figure 11. The 3D form of the nanopteron soliton. 


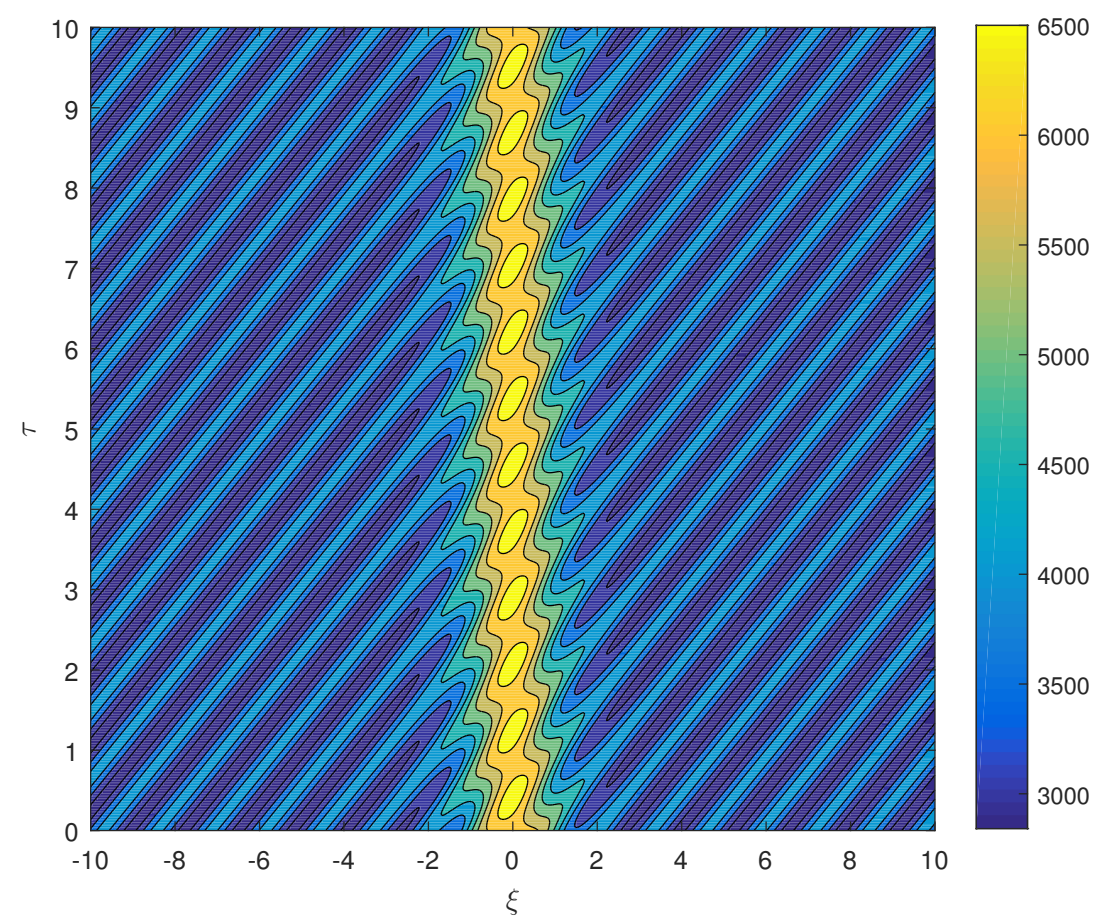

Figure 12. The contour plot of $\phi_{1}$ given by (40).

\section{Critical Nonlinearity and MKdv Equation}

In the KdV equation derived in Equation (31) of Section 4, the nonlinear coefficient ' $A$ ' can vanish depending on the values of various parameters. In order to analyze this, we have plotted the form of ' $A$ ' as a function of $\beta=\left(\frac{n_{i 0}}{Z_{d} n_{d 0}}\right)$ depending on various values of the electron streaming velocities. In Figure 13 we have depicted this situation, where it is observed that, in presence of strong coupling, $A$ can vanish for a particular situation.

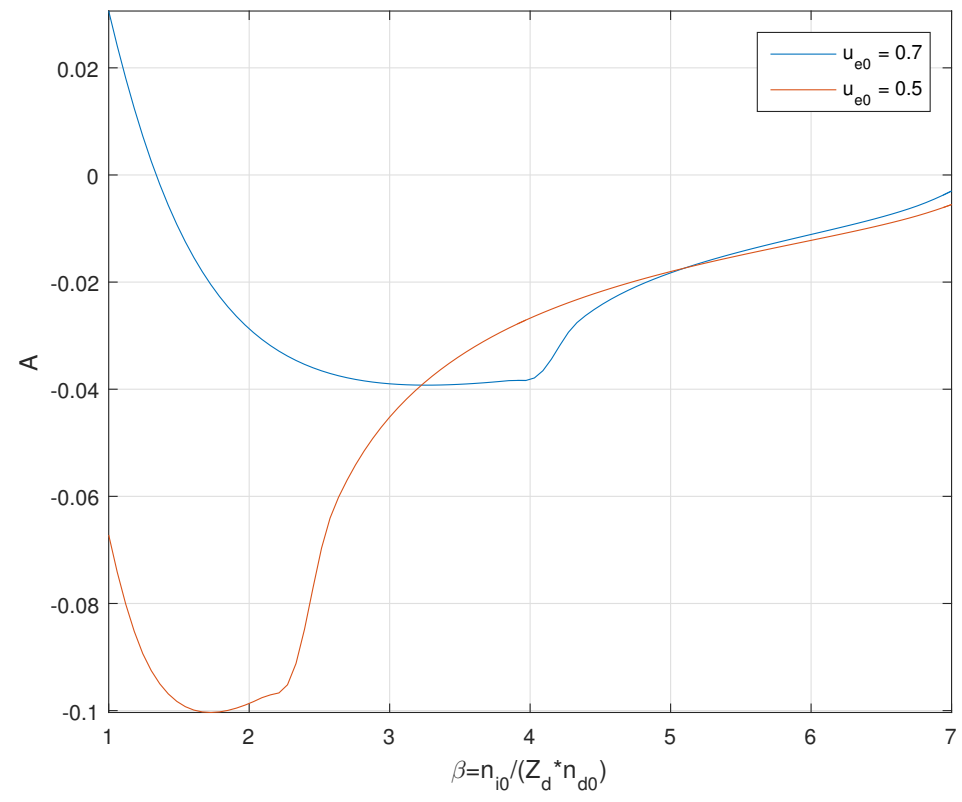

Figure 13. The variation of nonlinear coefficient $A$ with $\beta$.

In order to analyze such situations, we set the following.

$$
\left.\begin{array}{r}
\xi=\epsilon(x-\lambda t) \\
\tau=\epsilon^{3} t
\end{array}\right\}
$$


Since all the first order quantities remain the same. We only quote the second order and higher order perturbed quantities.

$$
\begin{gathered}
n_{e 2}=\frac{u_{e 2}}{\lambda-u_{e 0}}+\frac{u_{e 1} n_{e 1}}{\lambda-u_{e 0}} \\
u_{e 2}=-\frac{\alpha_{3} / Z_{d}}{\lambda-u_{e 0}} \phi_{2}+\frac{u_{e 1}^{2} / 2}{\lambda-u_{e 0}} \\
n_{d 2}=\frac{u_{d 2}}{\lambda}+\frac{u_{e 1} n_{e 1}}{\lambda} \\
u_{d 2}=\frac{\phi_{2}}{\lambda}+\frac{d_{2}}{\lambda}+\frac{d_{1} n_{d 1}}{\lambda}+\frac{d_{0} n_{d 2}}{\lambda}-\frac{u_{d 1}^{2}}{\lambda} \\
\mu_{1} n_{e 2}+n_{d 2}+\beta S \phi_{2}-\beta S^{2} \phi_{1}^{2}=0
\end{gathered}
$$

which provides the following.

$$
\phi_{2}=\frac{\chi_{2}}{\chi_{1}} \phi_{1}^{2}
$$

$\chi_{1}=-\frac{\alpha_{1} \mu_{1}}{Z_{d}\left(\lambda-u_{e 0}\right)^{2}}+\frac{\lambda\left(d_{22}-1\right)}{\left[\lambda^{2}-\left(d_{0}+d_{11}\right)\right]}+\beta$

$\chi_{2}=\frac{3 \mu_{1}\left(\alpha_{1} / Z_{d}\right)^{2}}{2\left(\lambda-u_{e 0}\right)^{4}}+\frac{\lambda\left(d_{23}+d_{11}\right)\left(d_{12}-1\right)^{2}}{\left[\lambda^{2}-\left(d_{0}+d_{11}\right)\right]^{3}}+\frac{\lambda\left(d_{12}-1\right)\left(d_{12}+d_{24}\right)}{\left[\lambda^{2}-\left(d_{0}+d_{11}\right)\right]^{2}}+\frac{\left(d_{12}-1\right)^{2}}{\left[\lambda^{2}-\left(d_{0}+d_{11}\right)\right]^{2}}+\frac{\lambda d_{25}}{\left[\lambda^{2}-\left(d_{0}+d_{11}\right)\right]}-\beta$

However, from the next higher order terms in $\epsilon$, we obtain the following:

$$
\begin{gathered}
-\left(\lambda-u_{e 0}\right) \frac{\partial n_{e 3}}{\partial \xi}+\frac{\partial n_{e 1}}{\partial \tau}+\frac{\partial u_{e 3}}{\partial \xi}+\frac{\partial}{\partial \xi}\left(n_{e 1} u_{e 2}\right)+\frac{\partial}{\partial \xi}\left(n_{e 2} u_{e 1}\right)=0 \\
-\lambda \frac{\partial n_{d 3}}{\partial \xi}+\frac{\partial n_{e 1}}{\partial \tau}+\frac{\partial u_{d 3}}{\partial \tau}+\frac{\partial}{\partial \xi}\left(n_{d 1} u_{d 2}\right)+\frac{\partial}{\partial \xi}\left(n_{d 2} u_{d 1}\right)=0 \\
\frac{\partial u_{e 1}}{\partial \tau}-\left(\lambda-u_{e 0}\right) \frac{\partial u_{e 3}}{\partial \xi}+\frac{\partial}{\partial \xi}\left(u_{e 1} u_{e 2}\right)=\alpha_{1} H^{2} \frac{\partial^{3} n_{e 1}}{\partial \xi^{3}}+\frac{\alpha_{1}}{Z_{d}} \frac{\partial \phi_{3}}{\partial \xi} \\
\frac{\partial u_{d 1}}{\partial \tau}-\lambda \frac{\partial u_{d 3}}{\partial \xi}+\frac{\partial}{\partial \xi}\left(u_{d 1} u_{d 2}\right)=\alpha_{1} H^{2} \frac{\partial^{3} n_{d 1}}{\partial \xi^{3}}+ \\
\frac{\alpha_{1}}{Z_{d}} \frac{\partial \phi_{3}}{\partial \xi}-\frac{1}{Z_{d}} \frac{\partial d_{3}}{\partial \xi}-\frac{\partial}{\partial \xi}\left(d_{2} n_{d 1}\right)-\frac{\partial}{\partial \xi}\left(d_{1} n_{d 2}\right)- \\
\left(\lambda-\frac{d_{0}}{\lambda}\right) \frac{\partial u_{d 3}}{\partial \xi}-\frac{d_{0}}{\lambda}\left\{\frac{\partial n_{d 1}}{\partial \tau}+\frac{\partial}{\partial \xi}\left(n_{d 1} n_{d 2}\right)+\frac{\partial}{\partial \xi}\left(n_{d 2} u_{d 1}\right)\right\} \\
\frac{\partial^{2} \phi_{1}}{\partial \xi^{2}}=\mu_{1} n_{e 3}+n_{d 3}+\beta \phi_{3}-2 \beta \phi_{1} \phi_{2}
\end{gathered}
$$

from which we can deduce

$$
\frac{\partial \phi_{1}}{\partial \tau}+A \frac{\partial}{\partial \xi}\left(\phi_{1} \phi_{2}\right)+A^{\prime} \frac{\partial}{\partial \xi}\left(\phi_{1}^{3}\right)+B \frac{\partial^{3} \phi_{1}}{\partial \xi^{3}}=0
$$

which when $A \rightarrow 0$, it reduces to the M-KdV equation. The expression of the variable ' $d_{3}$ ' in Equation (48) is given as per Reference [24]. It is well known that the M-KdV equation also supports soliton solutions. However, here we found a new type of solution that is both solitonic and periodic in nature. For that we set the following:

$$
\phi_{1}(\xi, \tau)=U(s)
$$


where $s=\xi+\omega \tau$. The second step is to choose $U(s)$ as follows:

$$
\begin{aligned}
& U(s)=g_{0}+ \\
& \sum_{l=1}^{M}\left(\frac{z(s)}{1+\left[z(s)^{2}\right]}\right)^{(l-1)}\left(g_{l} \frac{z(s)}{1+[z(s)]^{2}}+f_{l} \frac{1-[z(s)]^{2}}{1+[z(s)]^{2}}\right)
\end{aligned}
$$

where $f_{l}, g_{l}$ are constants to be determined and $z(s)$ can be obtained as a solution of the following.

$$
\left(\frac{d z(s)}{d s}\right)^{2}=a+b z(s)^{2}+c z(s)^{4}
$$

Several solutions of (53) are given in Reference [17], from which we have the following:

$$
\phi_{1}(\xi, \tau)= \pm \sqrt{\frac{3 B}{2 R}} \mp \frac{\sqrt{6 B}}{R \sqrt{B / R}} \frac{1-c n^{2}(\eta, \sqrt{3} / 2)}{1+c n^{2}(\eta, \sqrt{3} / 2)}
$$

where $\eta=\xi-\frac{5 B}{2} \tau$.

On the other hand, for $\eta=\xi+\frac{5 B}{2} \tau$, we can obtain the following.

$$
\phi_{1}(\xi, \tau)= \pm \sqrt{-\frac{3 B}{2 R}} \mp \frac{\sqrt{6 B}}{R \sqrt{-3 B / 2 R}} \frac{1-c n^{2}(\eta, 1 / 2)}{1+c n^{2}(\eta, 1 / 2)}
$$

Here, ' $c n^{\prime}$ is the Jacobi elliptic Cosine function, $R=\frac{3 \chi_{2}}{2 \chi_{1}} A+3 A^{\prime}$, where $A$ and $A^{\prime}\left(=A_{1}^{\prime} / A_{2}\right)$ are given in Appendix $\mathrm{A}$, and $\chi_{1}$ and $\chi_{2}$ are given after Equation (44). A diagrammatic representation of $A^{\prime}$ is given in Figure 14, while the graphical representation of the solution of Equation (54) is given in Figures 15 and 16, which shows both a soliton and periodic behaviour. We have the used the same plasma parameters as in Section 4.1 for plotting these figures too.

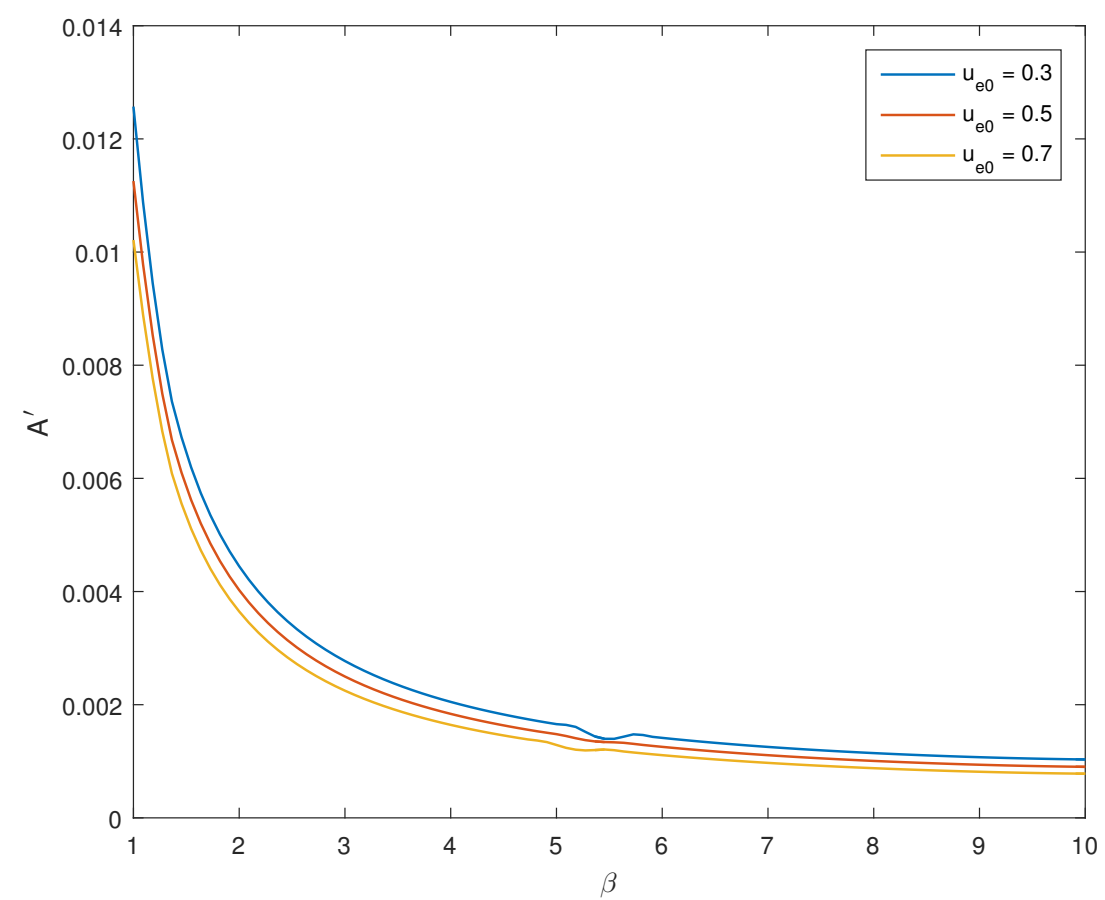

Figure 14. The variation of the nonlinear coefficient $\left(A^{\prime}\right)$ in the case of a modified KdV equation with $\beta\left(=n_{i 0} /\left(Z_{d} n_{d 0}\right)\right)$. 


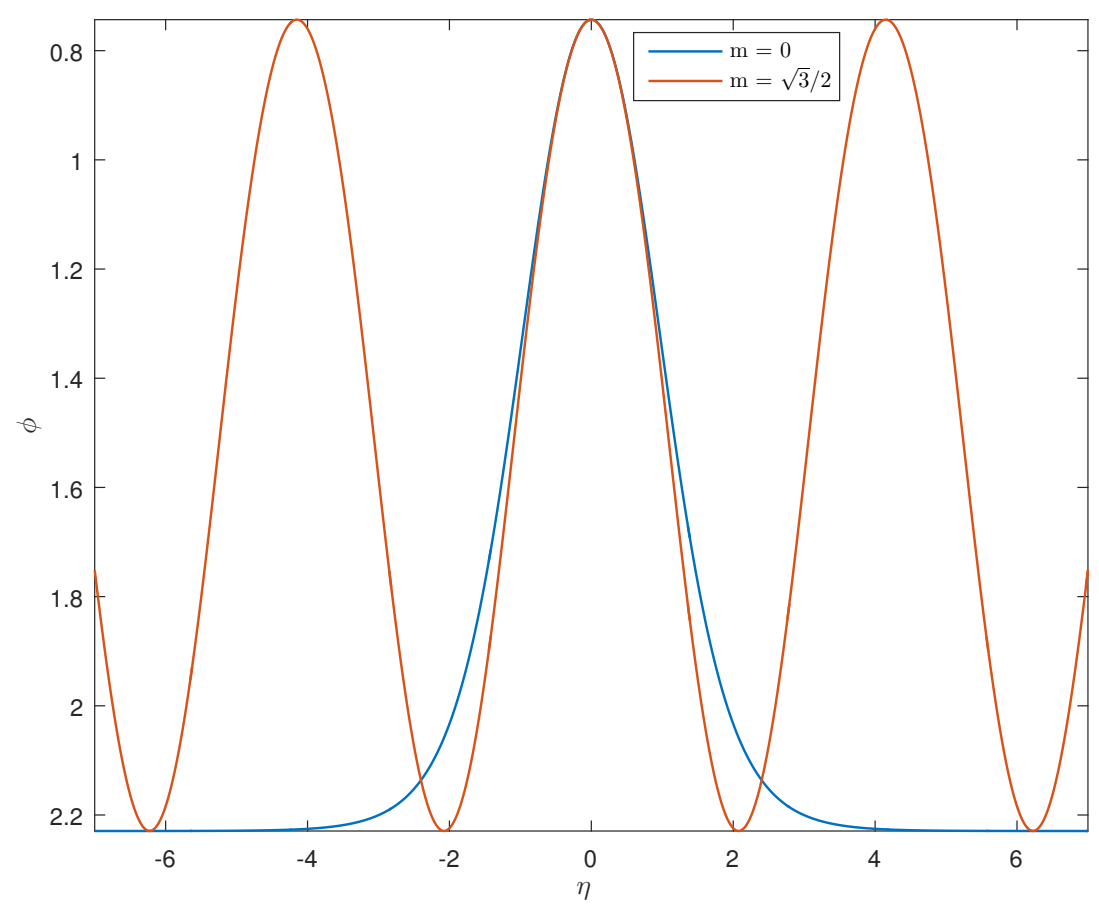

Figure 15. The graphical representation of quasi-soliton for MKdV equation for which its analytical form is represented by Equation (54).

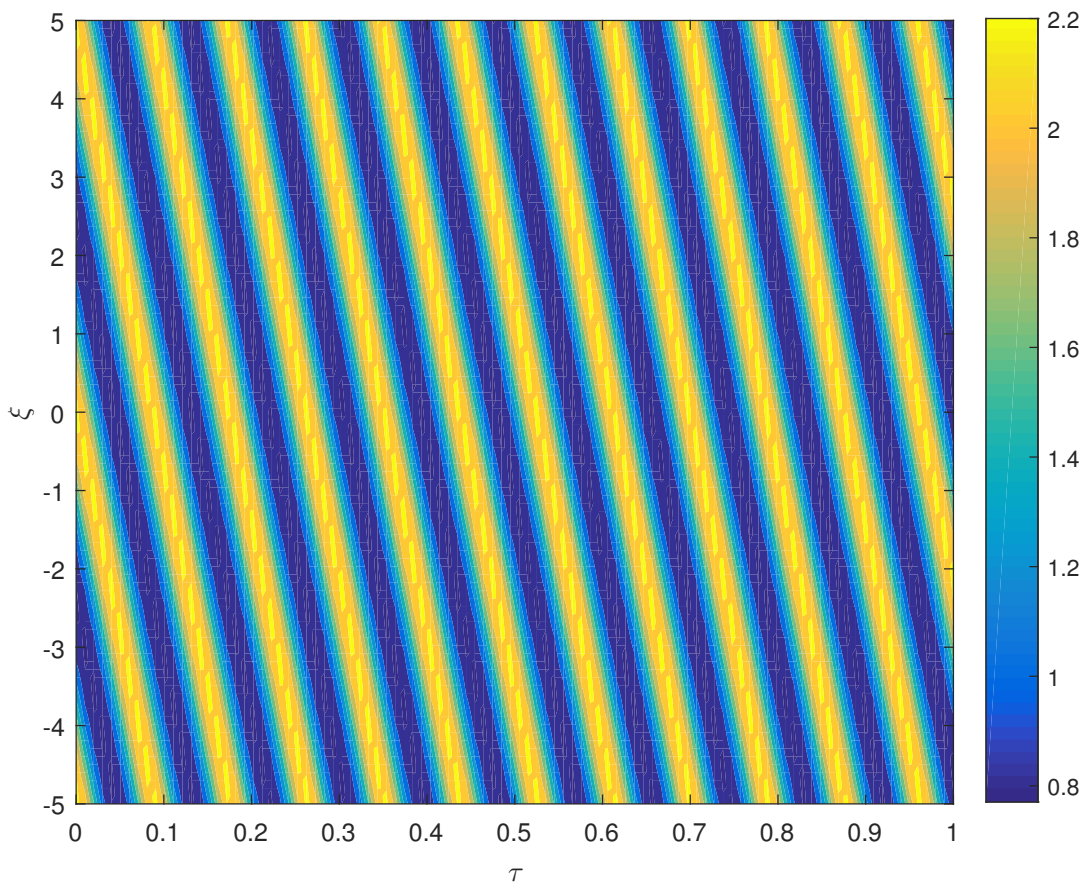

Figure 16. The contour plot of quasi-soliton for MKdV equation.

\section{Conclusions}

In our above analysis, we have shown how an electron streaming in a quantum strongly coupled dusty plasma can generate both 'slow' and 'fast' modes in the plasma. Earlier this type of phenomenon was also studied by Shukla et al. [25] but that model did not take into account the quantum effects or strong coupling but the effect of the magnetic field. Though they have considered the streaming of ions, in our case the streaming of electrons have been taken into account since the electrons are light compared to that of ions or dust. These are then manifested as some nonlinear type of excitation in the ensuing 
$\mathrm{KdV}$ equations, which are a new type of solitonic solutions with periodic tails. Nowadays, such solutions are called 'nanopteron' solitons. Due to the role of various parameters and the electron streaming velocity, a critical situation can sometimes be arrived at where the nonlinear coefficient of the ' $K d V^{\prime}$ ' equation tends to zero. Here, we then take recourse to a modified scaling and the perturbation procedure, to arrive at the 'MKdV' equation. It is observed that the MKdV equation can also have a new type of nonlinear excitation, which reflects both a solitonic and periodic nature at the same time. The whole process is surely influenced by the strength of the strong coupling and other parameters. It may be added that the nature of the strong coupling force in the form of the potential was discussed in References [23,26] and calculated in detail in Reference [23].

Author Contributions: S.C. and A.R.C. contributed equally to the paper. All authors have read and agreed to the published version of the manuscript.

Funding: There is no funding to report.

Institutional Review Board Statement: Not applicable.

Informed Consent Statement: Not applicable.

Data Availability Statement: No data is associated with this paper.

Conflicts of Interest: There is no conflict of interest.

\section{Abbreviations}

The following abbreviations are used in this manuscript:

RPT Reductive Perturbation Technique;

KdV Korteweg-deVries;

MKdV Modified Korteweg-deVries.

\section{Appendix A}

$$
\begin{aligned}
& A_{1}=\left(\left(\left(d_{12}+1\right)^{2}\right)\left(Z_{d}^{2}\right)\left(\left(\lambda-u_{e 0}\right)^{2}\right)\right) /\left(\left(Z_{d} \lambda^{2}-\left(d_{0}+d_{11}\right)\right)^{2}\right) \\
& -\left(2\left(d_{12}+1\right) Z_{d}\left(\lambda-u_{e 0}\right)^{2}\right) /\left(Z_{d} \lambda^{2}-\left(d_{0}+d_{11}\right)\right)+ \\
& 2\left(d_{23}\left(\left(d_{12}+1\right)^{2}\right)\left(Z_{d}^{2}\right)\left(\lambda-u_{e 0}\right)^{2}\right) /\left(\lambda ^ { 2 } \left(Z_{d} \lambda^{2}-\right.\right. \\
& \left.\left.\left(d_{0}+d_{11}\right)\right)^{2}\right)+\left(2 d_{24}\left(d_{12}+1\right) Z_{d}\left(\lambda-u_{e 0}\right)^{2}\right) /\left(Z_{d} \lambda-\left(d_{0}+d_{11}\right)\right)+ \\
& 2 d_{25}\left(\left(\lambda-u_{e 0}\right)^{2}\right)+\left(\left(2 d_{11}\left(\lambda-u_{e 0}\right)^{2}\right) /\left(Z_{d} \lambda^{2}\right)\right)\left(\left(d_{12}+1\right) Z_{d} /\left(\lambda^{2}-\right.\right. \\
& \left.\left.\left(d_{0}+d_{11}\right)\right)\right)^{2}+ \\
& \left(d_{12} / Z_{d}\right)\left(\left(d_{12}+1\right) Z_{d}\left(\lambda-u_{e 0}\right)^{2}\right) /\left(\lambda\left(Z_{d} \lambda^{2}-\left(d_{0}+d_{11}\right)\right)\right)+ \\
& \left(\mu_{1} / Z_{d}\right)\left(Z_{d} \lambda^{2}+\left(d_{0}+d_{11}\right)\right)\left(\left(\alpha_{3} /\left(Z_{d}\left(\lambda-u_{e 0}\right)\right)\right)^{2}-\right. \\
& \left.\alpha_{3} /\left(Z_{d}\left(\lambda-u_{e 0}\right)\right)\right)-2 \beta\left(( 1 / Z _ { d } ) \left(Z_{d} \lambda^{2}+\right.\right. \\
& \left.\left.\left(d_{0}+d_{11}\right)\right)\left(\lambda-u_{e 0}\right)^{2}\right) \\
& \quad A_{2}=-\left(1 / Z_{d}\right)\left(\left(Z_{d} \lambda^{2}+(d 0+d 11)\right)\left(\lambda-u_{e 0}\right)^{2}\right)+ \\
& \quad \alpha_{1}\left(H^{2}\right)\left(\left(\left(d_{12}+1\right) Z_{d}\right) /\left(Z_{d} \lambda^{2}-\right.\right. \\
& \left.\left.\quad\left(d_{0}+d_{11}\right)\right)\right)(1 / \lambda)-\alpha_{1}\left(H^{2}\right) a l p h a_{1} /\left(\left(\lambda-u_{e 0}\right)^{2}\right)
\end{aligned}
$$




$$
\begin{aligned}
& A_{1}^{\prime}=\left(3\left(\lambda^{2}\right) Z_{d}\left(d_{12}+Z_{d}\right)^{2}\right) /\left(\left(\left(\lambda^{2}\right) Z_{d}-\left(d_{0}+d_{11}\right)\right)^{2}\right)+ \\
& \left(2 d_{23}\left(d_{12}+Z_{d}\right)^{2}\right) /\left(\left(\left(\lambda^{2}\right) Z_{d}-\left(d_{0}+d_{11}\right)\right)^{2}\right)+ \\
& \left(2 d_{24}\left(d_{12}+Z_{d}\right)\right) /\left(\left(\left(\lambda^{2}\right) Z_{d}-\left(d_{0}+d_{11}\right)\right)^{2}\right)+ \\
& d_{25} /\left(\left(\left(\lambda^{2}\right) Z_{d}-\left(d_{0}+d_{11}\right)\right)^{1}\right)+ \\
& \left(2 d_{11}\left(d_{12}+Z_{d}\right)^{2}\right) /\left(\left(\left(\lambda^{2}\right) Z_{d}-\left(d_{0}+d_{11}\right)\right)^{2}\right)+ \\
& d_{12}\left(d_{12}+Z_{d}\right) /\left(\left(\left(\lambda^{2}\right) Z_{d}-\left(d_{0}+d_{11}\right)\right)^{1}\right)- \\
& \left(3 \beta\left(\alpha_{1}^{2}\right)\left(b e t a^{2}\right)\right) /\left(\lambda^{4}\right)-\left(2 \beta\left(\alpha 4^{2}\right) /\left(\left(\lambda^{3}\right)\left(Z_{d}^{2}\right)\right)\right)+ \\
& \left(\left(\beta\left(\alpha_{1}\right)\right) /\left(\left(Z_{d}^{2}\right)\left(\lambda^{4}\right)\right)-\right. \\
& \left.2 \mu_{1}\right)\left(3\left(\lambda^{2}\right) Z_{d}\left(d_{12}+Z_{d}\right)^{2}\right) /\left(\left(\left(\lambda^{2}\right) Z_{d}-\left(d_{0}+d_{11}\right)\right)^{2}\right)+ \\
& \left(2 d_{23}\left(d_{12}+Z_{d}\right)^{2}\right) /\left(\left(\left(\lambda^{2}\right) Z_{d}-\left(d_{0}+d_{11}\right)\right)^{2}\right)+ \\
& \left(2 d_{24}\left(d_{12}+Z_{d}\right)\right) /\left(\left(\left(\lambda^{2}\right) Z_{d}-\left(d_{0}+d_{11}\right)\right)^{2}\right)+ \\
& d_{25} /\left(\left(\left(\lambda^{2}\right) Z_{d}-\left(d_{0}+d_{11}\right)\right)^{1}\right)+ \\
& \left(2 d_{11}\left(d_{12}+Z_{d}\right)^{2}\right) /\left(\left(\left(\lambda^{2}\right) Z_{d}-\left(d_{0}+d_{11}\right)\right)^{2}\right)+d_{12}\left(d_{12}+\right. \\
& \left.Z_{d}\right) /\left(\left(\left(\lambda^{2}\right) Z_{d}-\left(d_{0}+d_{11}\right)\right)^{1}\right)- \\
& \left(3 \beta\left(\alpha_{1}^{2}\right)\left(\beta^{2}\right)\right) /\left(\lambda^{4}\right)- \\
& \left(2 \beta\left(\alpha 4^{2}\right) /\left(\left(\lambda^{3}\right)\left(Z_{d}^{2}\right)\right)\right)+\left(\left(\beta\left(\alpha_{1}\right)\right) /\left(\left(Z_{d}^{2}\right)\left(\lambda^{4}\right)\right)-2 \mu_{1}\right)+ \\
& \left(2 d_{35}\left(d_{12}+Z_{d}\right)\right) /\left(\left(\left(\lambda^{2}\right) Z_{d}-\left(d_{0}+d_{11}\right)\right)^{2}\right)+ \\
& d_{36} /\left(\left(\left(\lambda^{2}\right) Z_{d}-\left(d_{0}+d_{31}\right)\right)^{1}\right) \\
& B_{1}=\left(\left(\left(d_{12}+1\right)^{2}\right)\left(Z_{d}^{2}\right)\left(\left(\lambda-u_{e 0}\right) .^{2}\right)\right) . /\left(\left(Z_{d} \lambda^{2}{ }^{2}-\left(d_{0}+d_{11}\right)\right)^{2}\right) \\
& -\left(2\left(d_{12}+1\right) Z_{d}\left(\lambda-u_{e 0}\right)^{2}\right) /\left(Z_{d} \lambda^{2}-\left(d_{0}+d_{11}\right)\right)+ \\
& 2\left(d_{23}\left(\left(d_{12}+1\right)^{2}\right)\left(Z_{d}^{2}\right)\left(\lambda-u_{e 0}\right)^{2}\right) /\left(\lambda^{2}\left(Z_{d} \lambda^{2}-\left(d_{0}+d_{11}\right)\right)^{2}\right)+ \\
& \left(2 d_{24}\left(d_{12}+1\right) Z_{d}\left(\lambda-u_{e 0}\right)^{2}\right) /\left(Z_{d} \lambda-\left(d_{0}+d_{11}\right)\right)+ \\
& 2 d_{25}\left(\left(\lambda-u_{e 0}\right)^{2}\right)+ \\
& \left(\left(2 d_{11}\left(\lambda-u_{e 0}\right)^{2}\right) /\left(Z_{d} \lambda^{2}\right)\right)\left(\left(d_{12}+1\right) Z_{d} /\left(\lambda^{2}-\left(d_{0}+d_{11}\right)\right)\right)^{2}+ \\
& \left(d_{12} / Z_{d}\right)\left(\left(d_{12}+1\right) Z_{d}\left(\lambda-u_{e 0}\right)^{2}\right) /\left(\lambda\left(Z_{d} \lambda^{2}-\left(d_{0}+d_{11}\right)\right)\right)+ \\
& \left(\mu_{1} / Z_{d}\right)\left(Z_{d} \lambda^{2}+\left(d_{0}+d_{11}\right)\right)\left(\left(\alpha_{1} /\left(Z_{d}\left(\lambda-u_{e 0}\right)\right)\right)^{2}-\right. \\
& \left.\alpha_{1} /\left(Z_{d}\left(\lambda-u_{e 0}\right)\right)\right)- \\
& 2 \mu_{1}\left(\left(1 / Z_{d}\right)\left(Z_{d} \lambda^{2}+(d 0+d 11)\right)\left(\lambda-u_{e 0}\right)^{2}\right)
\end{aligned}
$$

\section{References}

1. Horanyi, M.; Mendis, D.A. Trajectories of charged dust grains in the cometary environment. Astrophys. J. 1985, 294, 357-368. [CrossRef]

2. Horanyi, M.; Mendis, D.A. The dynamics of charged dust in the tail of comet Giacobini-Zinner. J. Geophys. Res. Space Phys. 1986, 91, 355-361. [CrossRef]

3. Whipple, E.C. Potentials of surfaces in space. Rep. Prog. Phys. 1981, 44, 1197. [CrossRef]

4. Hou, Y.W.; Ma, Z.W.; Yu, M.Y. The plasma wave echo revisited. Phys. Plasmas 2011, 18, 012108. [CrossRef]

5. Gardner, C.L. The quantum hydrodynamic model for semiconductor devices. SIAM J. Appl. Math. 1994, 54, 409-427. [CrossRef]

6. Haas, F. Quantum Plasmas; Springer: Berlin/Heidelberg, Germany, 2011; Volume 65. [CrossRef]

7. Haas, F.; Manfredi, G.; Feix, M. Multistream model for quantum plasmas. Phys. Rev. E 2000, 62, 2763-2772. [CrossRef] [PubMed]

8. Tsintsadze, L.N. Weibel instabilities in a completely degenerate electron fermi gas. Phys. Plasmas 2009, 16, 094507. [CrossRef]

9. Haas, F.; Eliasson, B. A new two-stream instability mode in magnetized quantum plasma. Phys. Scr. 2015, 90, 088005. [CrossRef]

10. Hasan, M.M.; Hossen, M.A.; Rafat, A.; Mamun, A.A. Effect of bohm quantum potential in the propagation of ion-acoustic waves in degenerate plasmas. Chin. Phys. B 2016, 25, 105203. [CrossRef]

11. Haas, F.; Manfredi, G.; Goedert, J. Nyquist method for Wigner-Poisson quantum plasmas. Phys. Rev. E $2001,64,026413$. [CrossRef] [PubMed] 
12. Bagchi, S.; Chowdhury, K.R.; Mishra, A.P.; Chowdhury, A.R. On the nonlinear excitation in self gravitating quantum dusty plasma. Int. J. Theor. Phys. 2009, 48, 1132-1141. [CrossRef]

13. Misra, A.P.; Chowdhury, A.R. Modulation of dust acoustic waves with a quantum correction. Phys. Plasmas 2006, 13, 072305. [CrossRef]

14. Ahmad, M.; Fahad, S.; Ur Rahman, W. Quantum kinetic dust acoustic waves in arbitrary degenerate quantum dusty plasma. Phys. Plasmas 2020, 27, 013701. [CrossRef]

15. Rao, N.N.; Shukla, P.K.; Yu, M.Y. Dust-acoustic waves in dusty plasmas. Planet. Space Sci. 1990, 38, 543-546. [CrossRef]

16. Merlino, R.L. Dust-Acoustic Waves: Visible Sound Waves. Am. Inst. Phys. 2009, 1188, 141-152. [CrossRef]

17. Haas, F.; Mahmood, S. Nonlinear ion-acoustic solitons in a magnetized quantum plasma with arbitrary degeneracy of electrons. Phys. Rev. E 2016, 94, 033212. [CrossRef] [PubMed]

18. Shukla, P.K.; Eliasson, B. Nonlinear aspects of quantum plasma physics. Physics-Uspekhi 2010, 53, 51. [CrossRef]

19. Ichimaru, S. Strongly Coupled Plasma Physics; Elsevier: Amsterdam, The Netherlands, 1990. [CrossRef]

20. Kaw, P.K.; Sen, A. Low frequency modes in strongly coupled dusty plasmas. Phys. Plasmas 1998, 5, 3552-3559. [CrossRef]

21. Gozadinos, G.; Ivlev, A.V.; Boeuf, J.P. A fluid model for colloidal plasmas under microgravity conditions. New J. Phys. 2003, 5, 32. [CrossRef]

22. Ikezi, H. Coulomb solid of small particles in plasmas. Phys. Fluids 1986, 29, 1764-1766. [CrossRef]

23. Cousens, S.E.; Sultana, S.; Kourakis, I.; Yaroshenko, V.V.; Verheest, F.; Hellberg, M.A. Nonlinear dust-acoustic solitary waves in strongly coupled dusty plasmas. Phys. Rev. E 2012, 86, 066404. [CrossRef] [PubMed]

24. Chaudhuri, S.; Chowdhury, A.R. KBM approach to dust acoustic envelope soliton in strongly coupled plasma. Chaos Solitons Fractals 2018, 109, 252-258. [CrossRef]

25. Shukla, N.; Shukla, P.K.; Brodin, G.; Stenflo, L. Ion streaming instability in a quantum dusty magnetoplasma. Phys. Plasmas 2008, 15, 044503. [CrossRef]

26. Yaroshenko, V.V.; Verheest, F.; Thomas, H.M.; Morfill, G.E. The Bohm sheath criterion in strongly coupled complex plasmas. New J. Phys. 2009, 11, 073013. [CrossRef] 